\title{
Radiologic Appearances in Fungal Rhinosinusitis
}

\author{
${ }^{1}$ Vicky S Khattar, ${ }^{2}$ Bachi T Hathiram
}

${ }^{1}$ Assistant Professor, Department of ENT and Head and Neck Surgery, TN Medical College and BYL Nair Charitable Hospital Mumbai Maharashtra, India

${ }^{2}$ Professor and Head, Department of ENT and Head and Neck Surgery, TN Medical College and BYL Nair Charitable Hospital Mumbai Maharashtra, India

Correspondence: Vicky S Khattar, Assistant Professor, Department of ENT and Head and Neck Surgery, TN Medical College and BYL Nair Charitable Hospital, C-7, Swati, Kalyan Complex, Panchmarg, Versova, Mumbai-400061, Maharashtra, India

\begin{abstract}
Radiological diagnosis forms an important tool in the armamentarium to diagnose and classify fungal rhinosinusitis. However, many times, the appearances may be deceptive; while on one hand in Allergic Fungal Rhinosinusitis the appearance is classical and considered one of the clinchers in the diagnosis of the condition, in invasive fungal rhinosinusitis (especially in the early phases), the appearances may range from a nonspecific sinusitis to even a 'apparently normal' scan! CT scan as well as MRI may at times complement each other, in order to aid the clinician arrive at a diagnosis. Nevertheless, despite the best radiologic modalities, a clinicoradiological correlation is a must to accurately diagnose this often deceptive condition called fungal rhinosinusitis.
\end{abstract}

Keywords: Imaging, computed tomography, paranasal sinuses, diagnosis.

\section{INTRODUCTION}

Fungal rhinosinusitis is a great imposter. While on one hand, certain radiologic appearances are characteristic for certain forms of the disease, yet there is a significant percentage of cases, wherein the best imaging techniques fail to clinch the diagnosis, and warrant the use of nonradiological diagnostic modalities.

Fungal rhinosinusitis is broadly classified into five types, namely:

1. Allergic fungal sinusitis (AFRS)

2. Fungal ball

3. Saprophytic colonization

4. Acute invasive

5. Chronic/indolent invasive

Each of the above mentioned categories present with certain typical radiological findings, and shall be discussed below.

\section{Allergic Fungal Rhinosinusitis (AFRS)}

It is a Gell and Coombs Type I mediated hypersensitivity reaction to an extramucosal fungal antigen, commonly seen in atopic individuals. It has a strong association with allergic rhinitis and asthma, and is associated with elevated serum IgE levels. Some have likened it to allergic broncho pulmonary aspergillosis (ABPA), and have postulated that the pathogenesis is a combination of Gell and Coombs Type I (atopic) and Type III (immune-complex mediated) hypersensitivity mechanisms.

\section{CT Findings}

Approximately half the cases ( $51 \%$ as studied by Mukherji et al, 1998) occur unilaterally (Fig. 1), and many others show asymmetric involvement (78\% as studied by Mukherji et al, 1998) by the disease on the two sides (Fig. 2). There is expansion of the involved sinuses with corresponding reduction in the space of the surrounding compartments such as the orbit (Fig. 3), which may be accompanied by remodelling (Fig. 3) or attenuation of the bony margins within the periphery of the lesion (Fig. 4). Several studies have quoted the incidence of bony erosion with spread of pathology into the adjacent anatomic areas as $20 \%{ }^{5,6}$ Whether this erosive effect represents a direct manifestation of the progression of the disease, or a different pathological entity within AFRS, has yet to be proven. ${ }^{10}$

AFRS is primarily caused by demitaceous fungi. The allergic mucin seen in such cases contains the maximum load of the fungal elements, which are known to have a great affinity for calcium, manganese, magnesium and other ferromagnetic substances. This is seen more so in cases which have not been treated with steroids. Since this mucin 


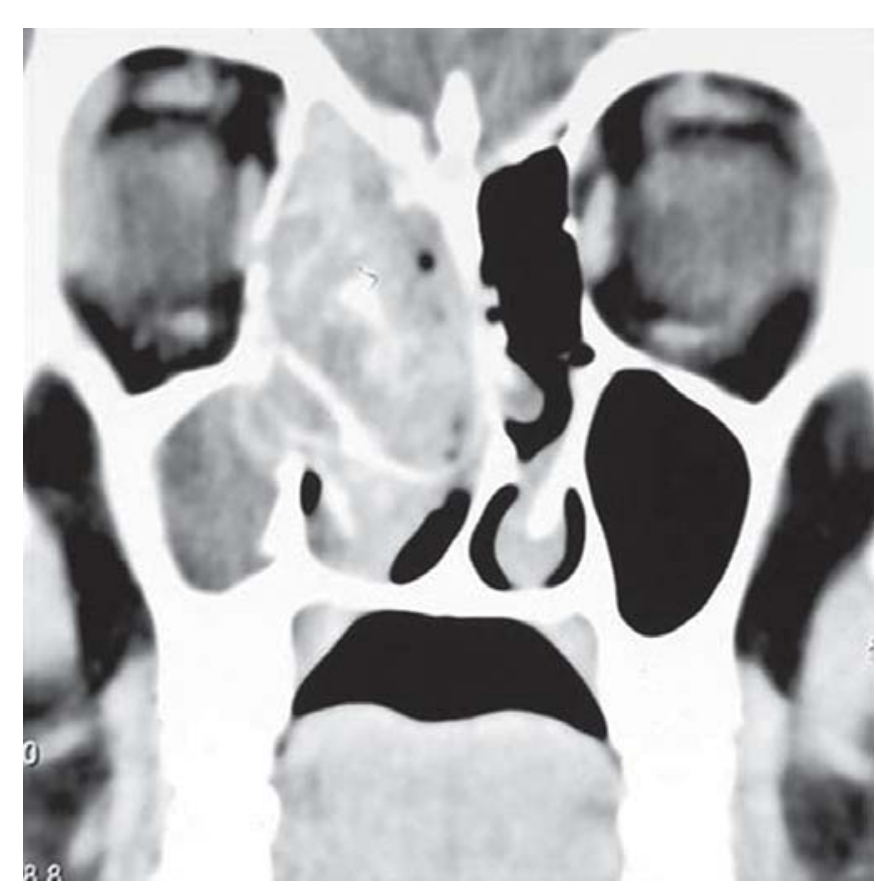

FIGURE 1: Coronal CT - PNS (computerized tomogram of the paranasal sinuses) showing unilateral (right sided) sinus opacification in a case of allergic fungal rhinosinusitis (AFRS)

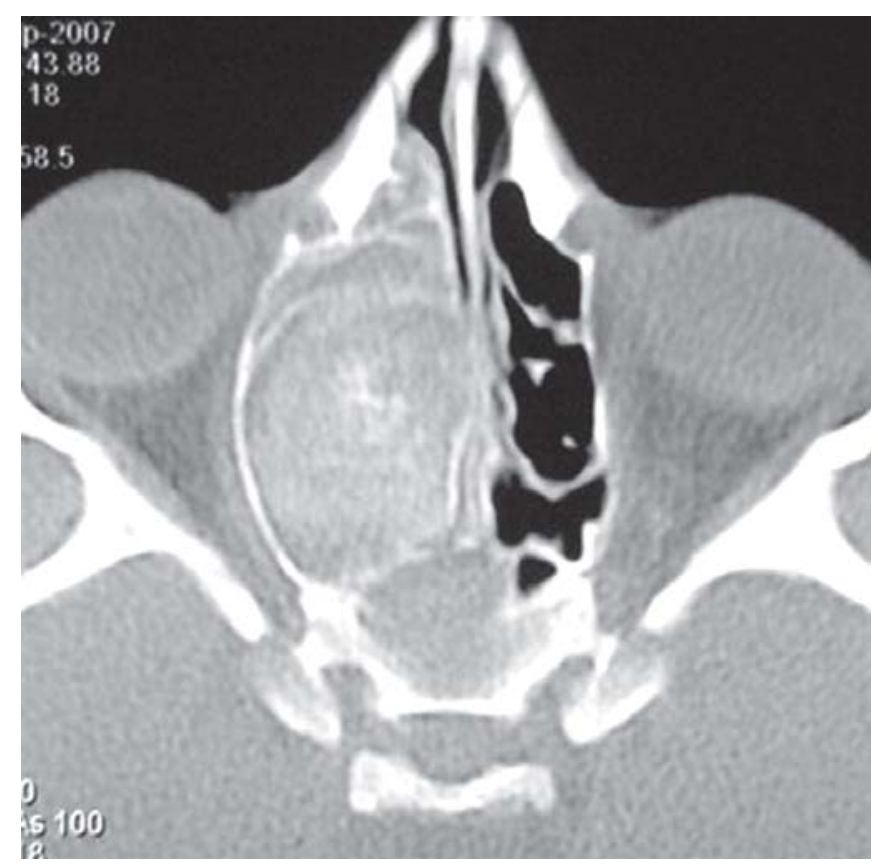

FIGURE 3: Axial CT - PNS showing expansion of the right sino-nasal compartment with corresponding compromise of the right orbital volume, with remodelling of the right lamina papyracea, as compared to the left side, in a case with right sided AFRS

is interspersed with fungal debris, concretions and polypoid mucosa, the unenhanced CT scan shows a characteristic heterogeneity of signal within the involved sinus, which has been given many names such as the 'starry-sky',

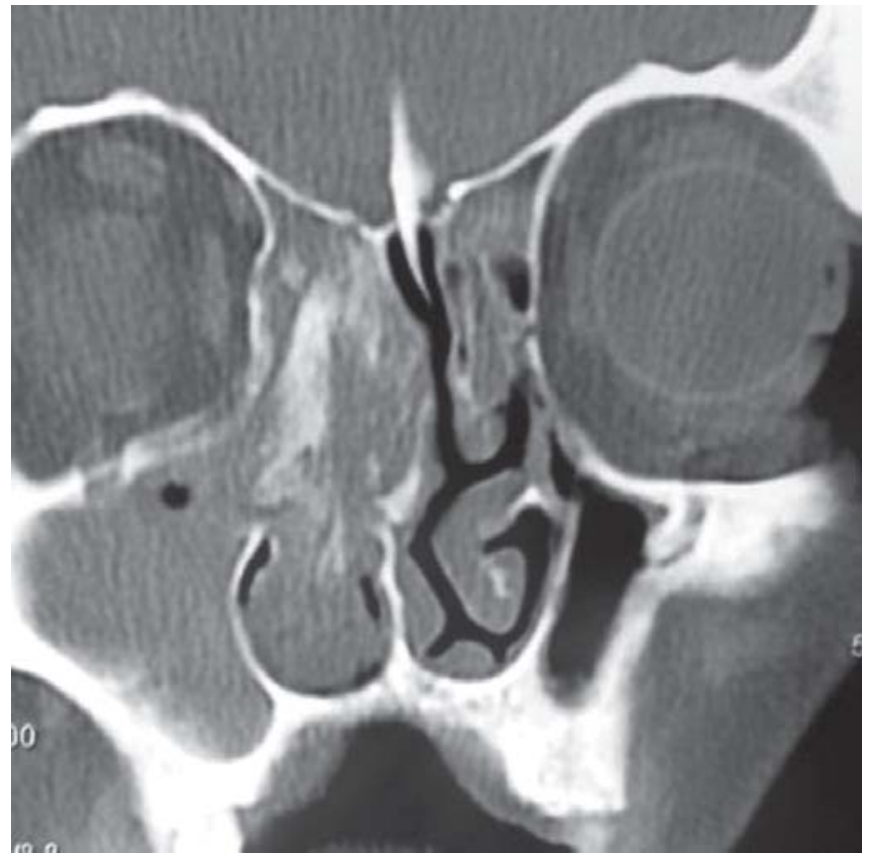

FIGURE 2: Coronal CT - PNS of a patient with AFRS showing extensive sinus involvement on the right side, with minimal disease on the left side

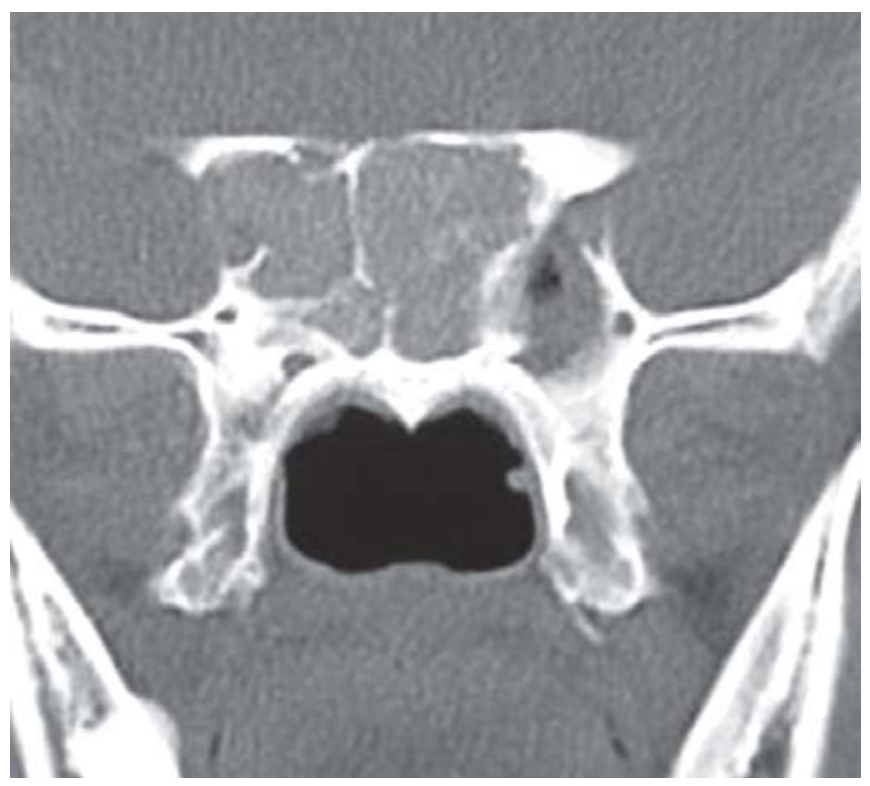

FIGURE 4: Coronal CT - PNS showing near complete attenuation of the lateral wall of the right sphenoid sinus, and partial attenuation of the roof of both sphenoid sinuses, in a case with bilateral AFRS

'ground-glass' or 'serpiginous' patterns, but commonly referred to as the 'double-density' sign, which by far, describes this typical radiologic appearance the best (Fig. 5). This is best appreciated in the soft tissue windows. ${ }^{4}$ 


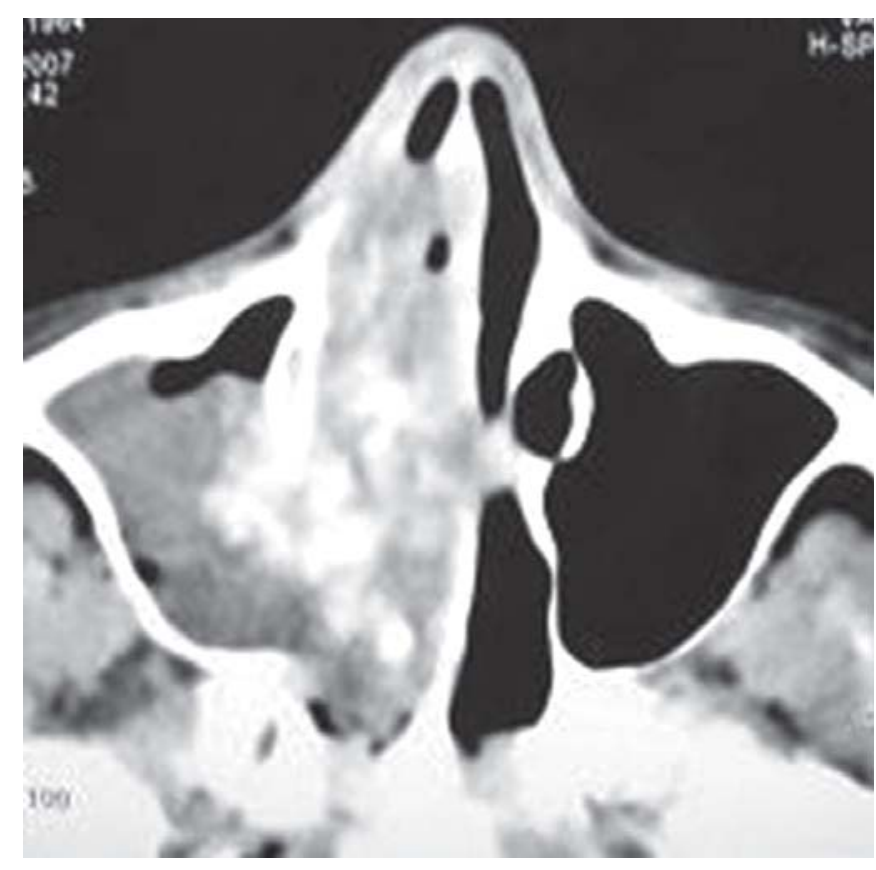

FIGURE 5: Axial CT - PNS showing the characteristic 'double-density' sign, in a patient with right sided AFRS

However, this ‘double density’ sign may also be seen with other forms of fungal sinusitis, and is usually caused by the dense inspissated eosinophil-rich extramucosal allergic mucin, which although found virtually in all cases of AFRS

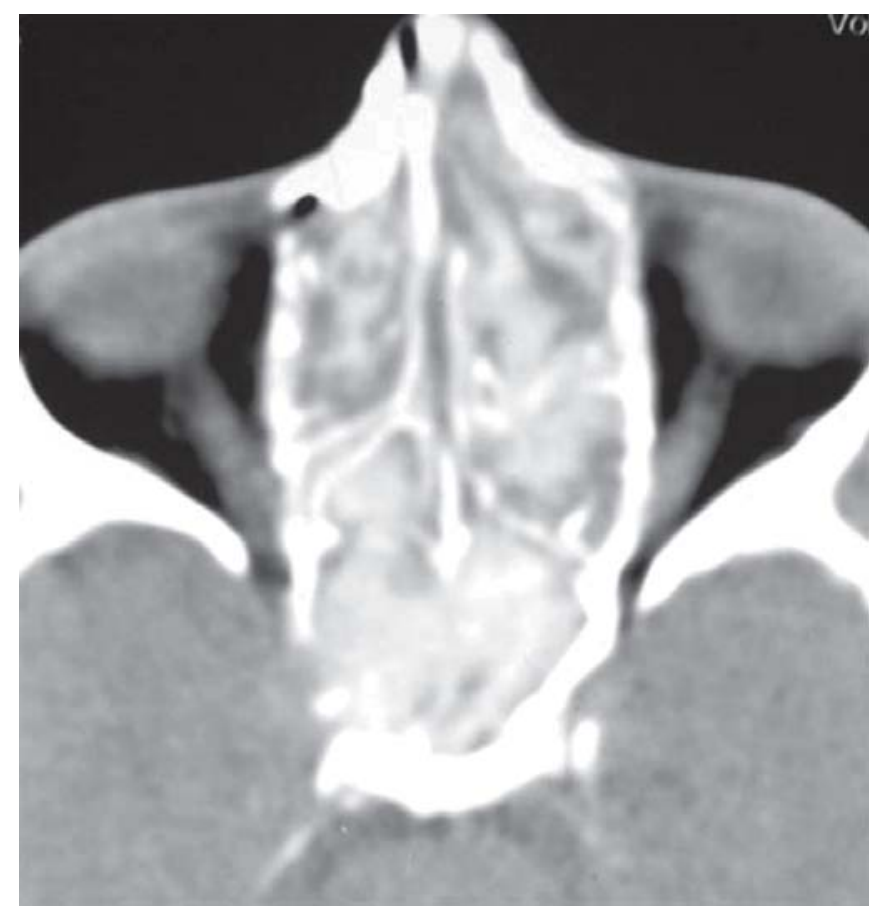

FIGURE 6: Preoperative, presteroid axial CT - PNS showing a complete 'white-out' appearance, with involvement of both sides sinonasal compartments during surgery, may also be seen in nonfungal hypertrophic sinus disease, such as the ASA triad (Samter's syndrome). Thus, according to Schubert (2004), the hyperattenuating signal on CT in AFS may be caused by both the presence of inspissated allergic mucin, and the fungal hyphae within it. Following steroid therapy, an initial complete 'white-out' appearance on CT scan (Fig. 6) changes to one with a decreased disease load (Fig. 7), leaving behind the medically irreversible changes which would warrant surgical intervention; it also allows for better denotation of bony landmarks, and helps in surgical road-mapping, besides the added advantage of reducing intraoperative bleeding. Thus it would not be unadvisable to say, that CT scans may be repeated after the preoperative steroid therapy, which in all probability would help in planning for a favorable surgical outcome.

There is a mention in literature of an entity called as Eosinophilic mucin sinusitis, which although being similar to allergic fungal sinusitis is many aspects, differs from it in the fact that it has a stronger correlation with asthma, is more commonly bilateral as compared to AFRS, and shows a greater percentage of eosinophilia within the mucin, with absence of fungal elements within the mucin.

\section{MR Findings}

The characteristic appearance is a iso or hypoattenuated signal on T1 weighted images, and a signal void on T2 weighted images in AFRS. The high protein and low water

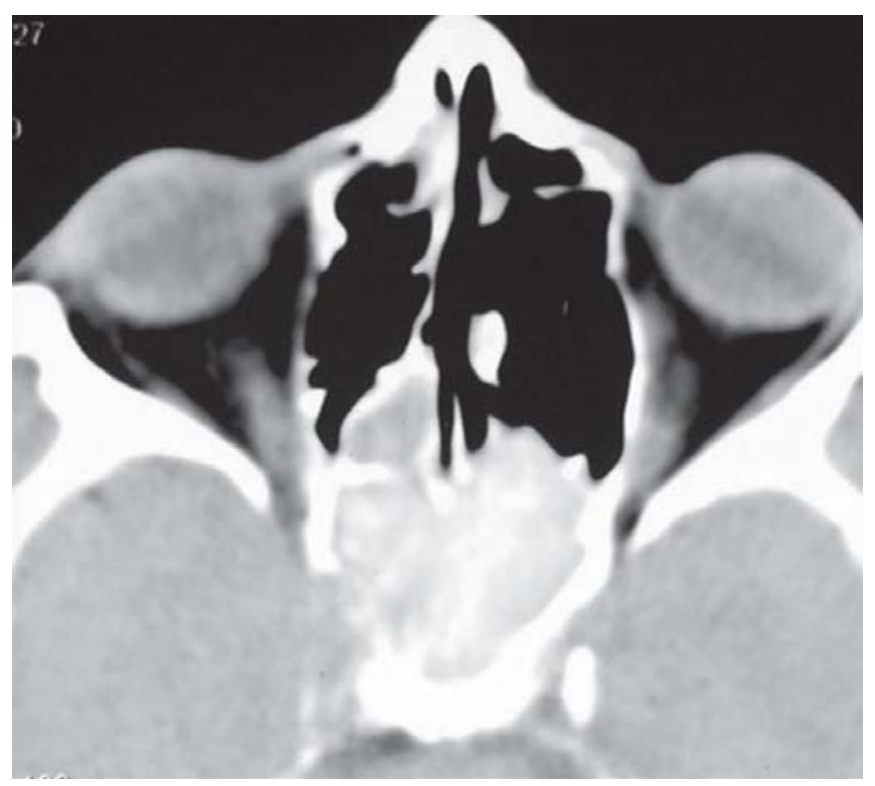

FIGURE 7: Preoperative, poststeroid axial CT - PNS of the same patient (Fig. 6) showing a dramatic reduction in the bulk of disease 


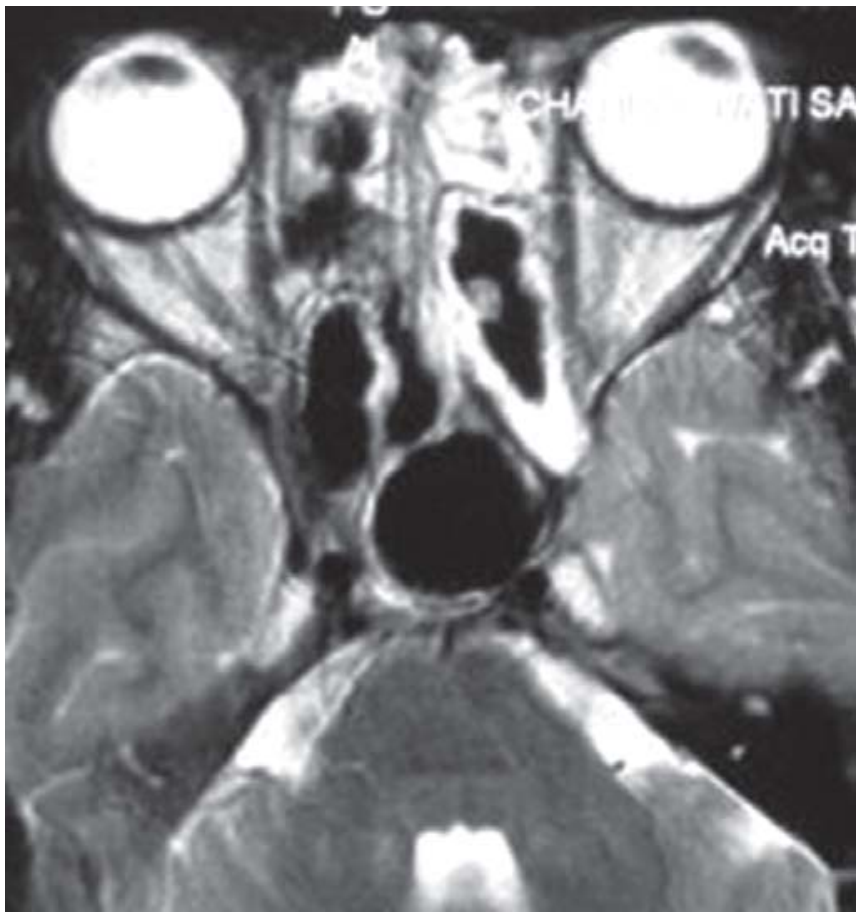

FIGURE 8: Axial T2 MRI - PNS in a case of bilateral AFRS, showing the characteristic features signal void in the involved sinuses, and hyperintense signals in the sinuses affected with secondary bacterial infection, or with edematous polypoid mucosa

concentration of allergic fungal mucin, coupled with the high water content within the surrounding edematous paranasal sinus mucosa, gives rise to the characteristic MR findings in AFRS, with this effect being more pronounced in the T2 images, due to a prolonged magnetic field relaxation time. ${ }^{7}$ The singal void on T2 images may mimic aeration. ${ }^{12}$ The surrounding inflamed polyps and mucosa are hyperintense on both T1 and T2 (Figs 8 and 9). ${ }^{13}$ MRI also helps in confirming the noninvasion of the dura and periorbita, which is typical of this disease. This may be correlated with the CT scan for better delineation of the disease (Figs 10 and 11).

\section{Fungal Ball}

It is an extramucosal collection of degenerating fungal hyphae, which have been packed into a mass, within a single sinus cavity. It is most commonly seen in the maxillary sinus (Fig. 12), followed by the sphenoid sinus, and less commonly in the ethmoid and frontal sinuses respectively. Usually there is a rim of reactive bony sclerosis around the opacified sinus (Fig. 12), due to an underlying indolent osteitis. There may be associated flocculent calcifications. ${ }^{9}$ These are typically seen in immuno-competent hosts, and can present with complaints related to expansion, or due to compression of surrounding anatomical structures (Fig. 13).

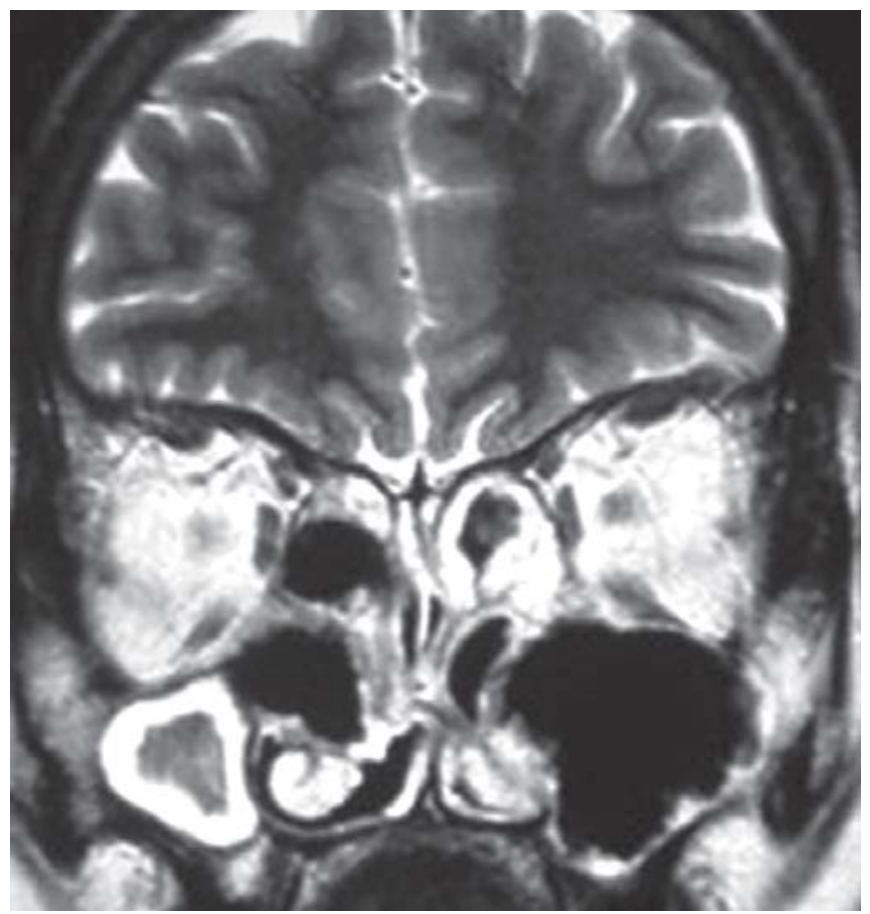

FIGURE 9: Coronal T2 MRI - PNS of the same patient (Fig. 8)

Fungal balls may cause expansion of the involved sinus by bony attenuation, due to an inflammatory reaction of the sinus mucosa, or a pressure effect. Sometimes, bone erosion may also be caused by hyphal proliferation. ${ }^{8}$ Fungal balls have the potential to progress to an invasive form, should the host immunity wane.

\section{Saprophytic Colonization}

Commonly seen in postoperative sinus cavities on crusts, their mere presence within the nose or sinuses does not indicate disease. Since there is no definitive pattern for their colonization, they would naturally not seem to have any particular radiologic appearance, and their diagnosis would essentially remain by endoscopic removal of crusts, and their evaluation with $\mathrm{KOH}$ mounts.

\section{Acute Invasive Fungal Rhinosinusitis}

Nothing causes a bigger radiologic diagnostic dilemma, than acute invasive fungal rhinosinusitis; notwithstanding the fact that the disease can be fatal, with rapid involvement of surrounding vital structures such as the eye and brain and thus requiring an early diagnosis; the sheer nonspecificity of the radiologic changes makes for a difficult diagnosis. The role of a high index of suspicion, coupled with a meticulous nasal endoscopic evaluation makes for an early accurate diagnosis, which may be life-saving. ${ }^{1}$ 


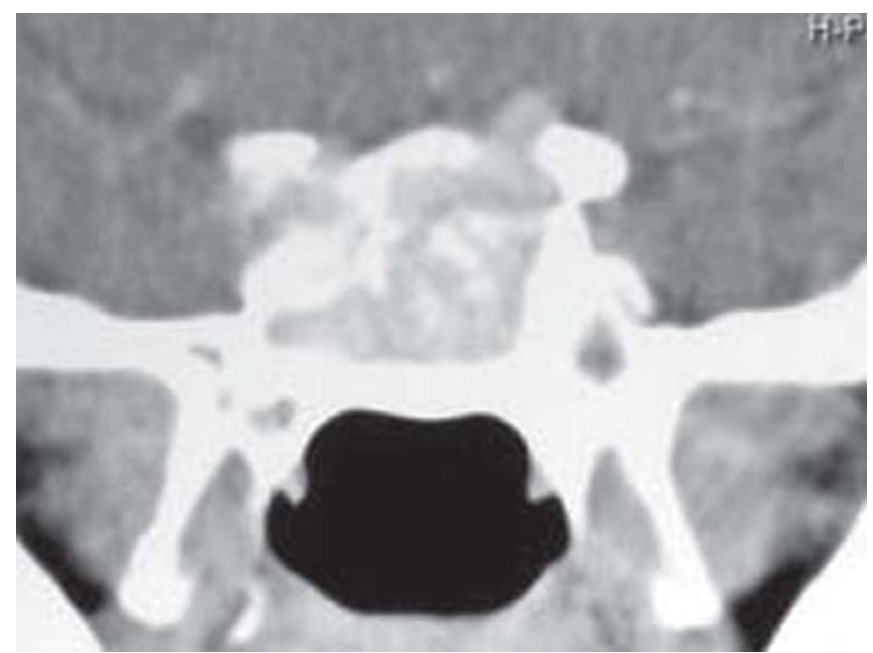

FIGURE 10: Coronal CT - PNS showing erosion of the roof of the left, and lateral wall of the right sphenoid sinus, in a case of bilateral AFRS

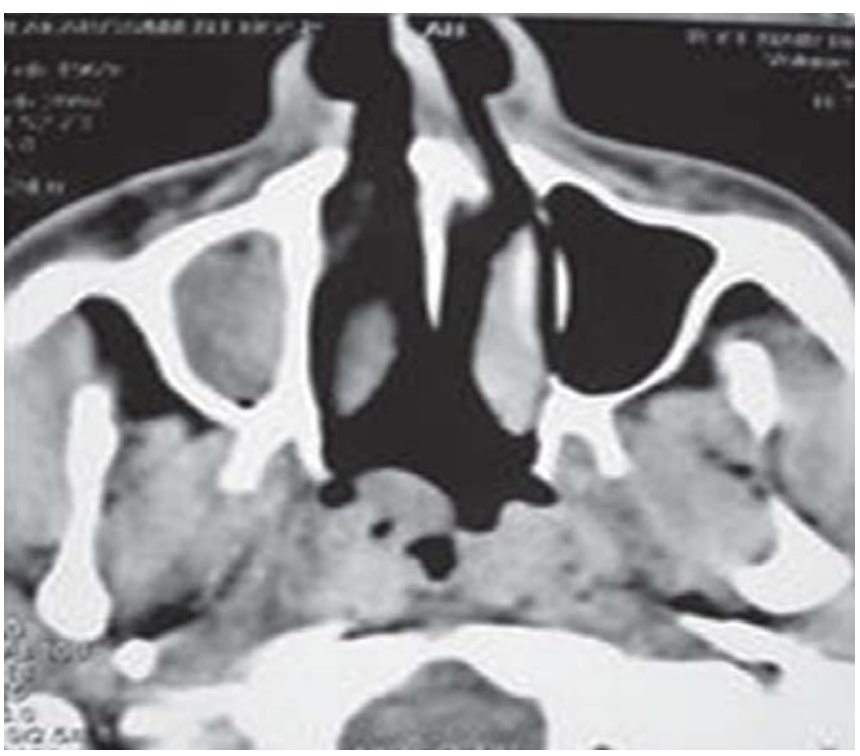

FIGURE 12: Axial CT - PNS showing an isolated right maxillary sinus opacification, with reactive sclerosis of the bony margins. The patient was found to have fungal mass within the right maxillary sinus on surgery, making the final diagnosis of a right fungal ball

CT scans (especially in the early course of the disease) usually show a normal study, or nonspecific mucosal thickening, indistinguishable from the appearance of bacterial/viral disease. ${ }^{2}$ The problem with diagnosis is compounded by the fact, that bone destruction and softtissue swelling occur in advanced disease (Figs 14 and 15). ${ }^{3}$

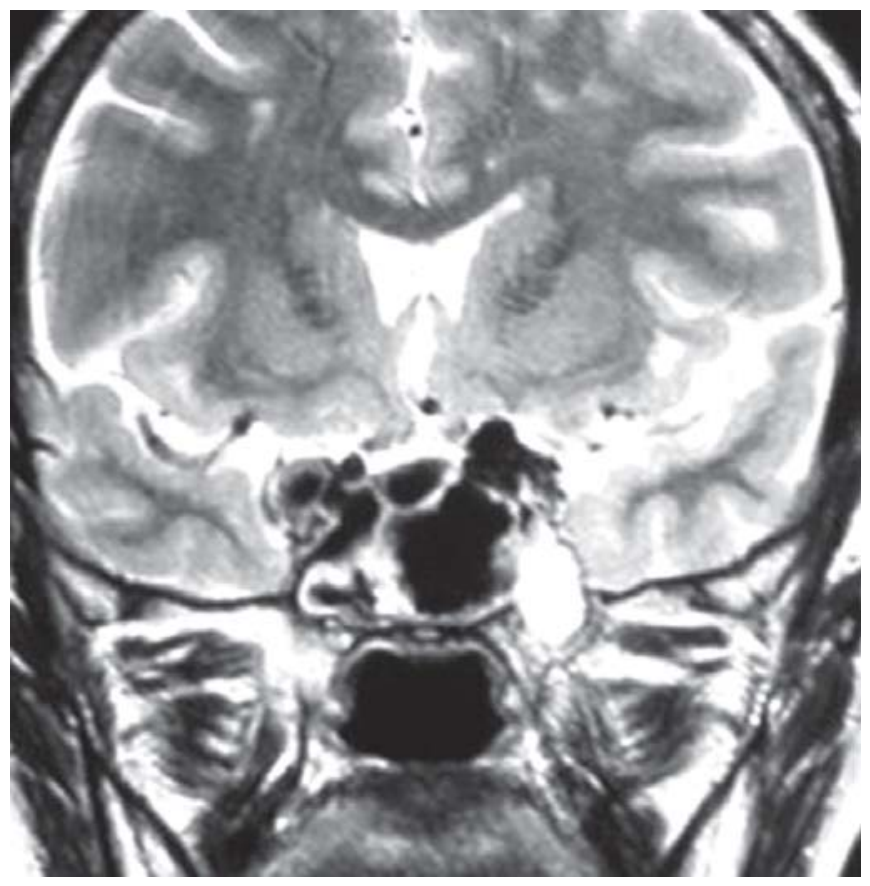

FIGURE 11: Coronal T2 MRI - PNS of the same patient (Fig. 10), depicting a thin rim of CSF (bright signal) around the uppermost extent of the disease (signal void), indicating its extradural nature

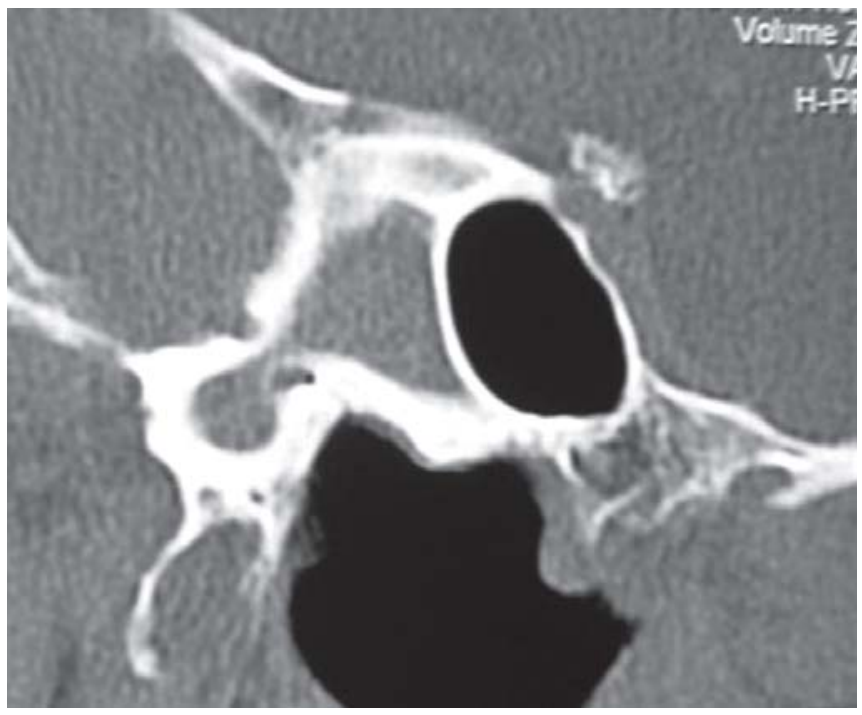

FIGURE 13: Coronal CT - PNS showing right sphenoid sinus opacification, with disease in the lateral recess of the right sphenoid sinus, in close proximity to the foramen rotundum and vidian canal. The patient presented with a right sided headache, which was relieved after an endoscopic evacuation of the right sphenoid sinus, with the $\mathrm{KOH}$ mount of the evacuated material showing fungal mycelia

\section{Chronic Invasive Fungal Rhinosinusitis}

This disease entity is characterized by a relatively slower progression than its acute fulminant counterpart, which is worsened by a high recurrence rate. The radiologic hallmark is bone destruction (Figs 16 and 17). ${ }^{3}$ There are however a 


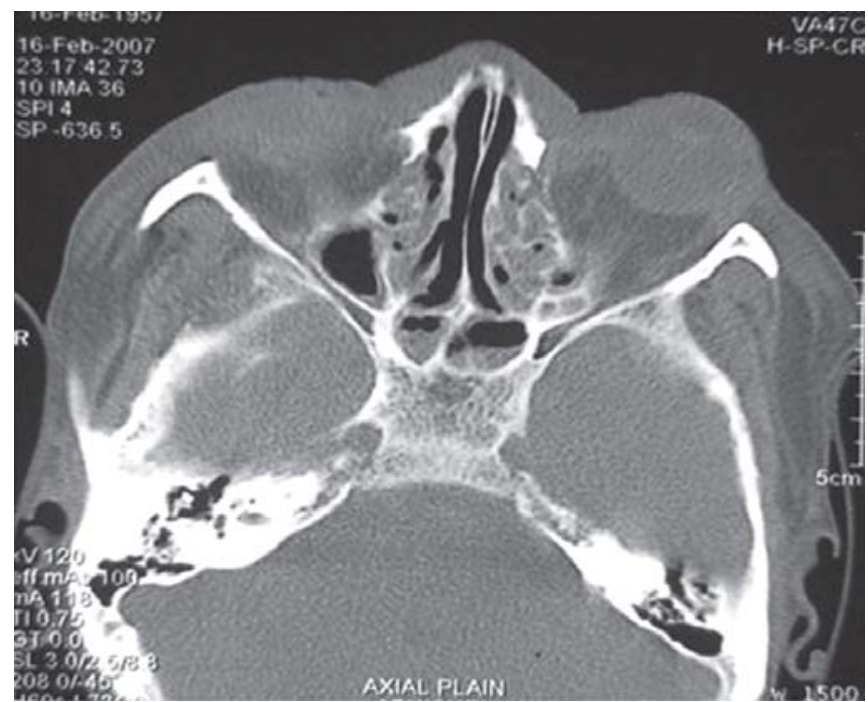

FIGURE 14: Axial CT - PNS of a patient with acute fulminant invasive fungal rhinosinusitis, with left proptosis. Note the non-specific appearance of the sinuses, similar to any bacterial/viral sinusitis. This was in stark contrast to the endoscopic image, which showed a complete obliteration of both nasal cavities with dense, black escharlike crusts (Fig. 15). Also evident is the left orbital proptosis

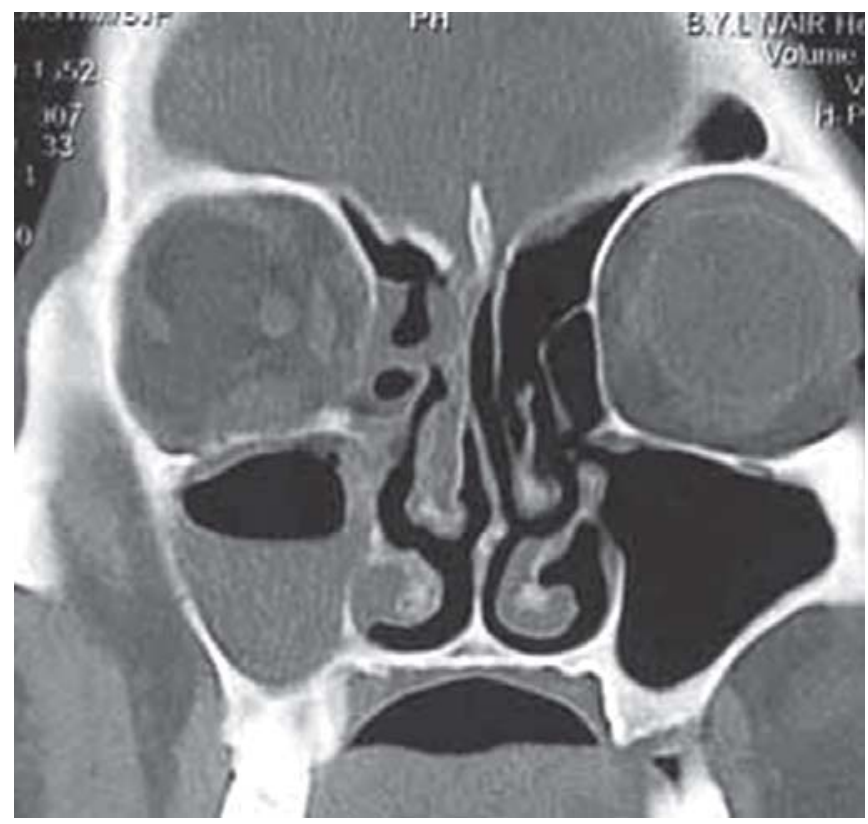

FIGURE 16: Coronal CT - PNS of a patient suffering from right sided chronic invasive fungal rhinosinusitis, with erosion of the lamina papyracea. Note again (as in Fig. 14), the non-specific appearance of the sinuses, mimicking a right sided acute maxillary and ethmoid sinusitis, with an air-fluid level in the right maxillary sinus. Also note the irregular appearance of the right middle turbinate (when compared to the left middle turbinate), which correlated well with the endoscopic appearance (Fig. 17)

few diagnostic and prognostic indicators for orbital involvement in invasive fungal rhinosinusitis. Features suggestive of orbital involvement include proptosis without

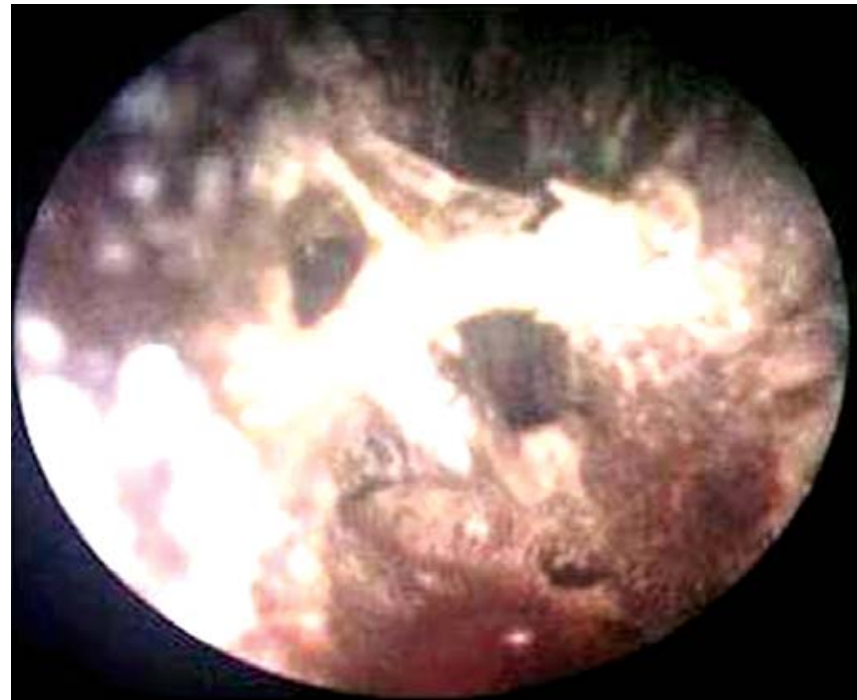

FIGURE 15: An endoscopic image of the left nasal cavity, showing complete obliteration with dense, black eschar-like crusts, in a patient with acute fulminant invasive fungal rhinosinusitis (CT scan in Fig. 14)

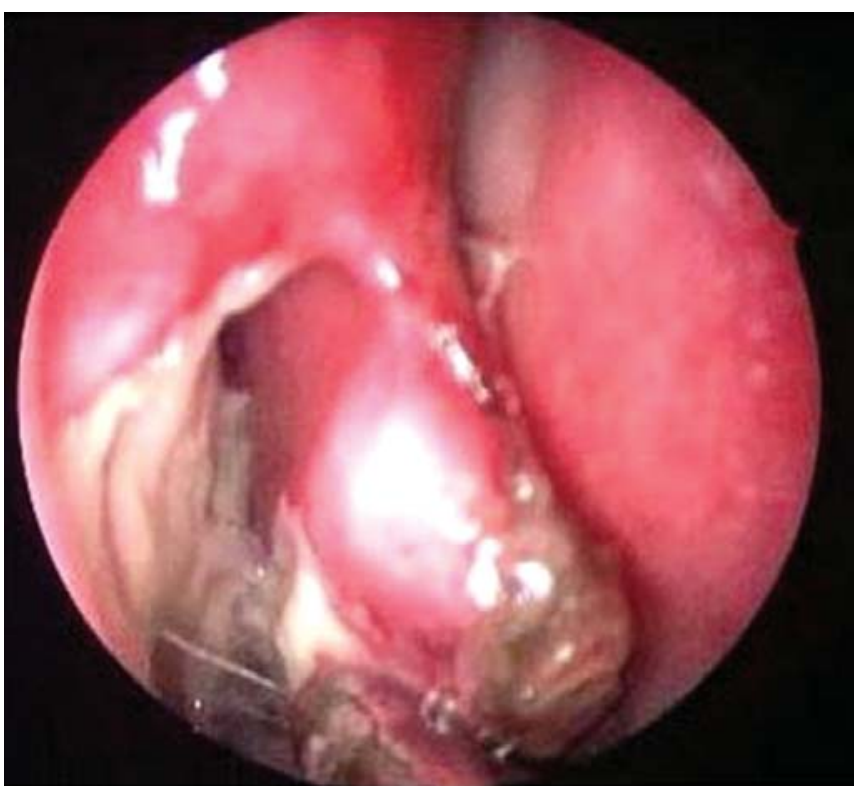

FIGURE 17: Endoscopic image of the right nasal cavity, showing blackening and crust formation of the right middle turbinate and middle meatus, the biopsy of which showed mycotic tissue invasion, and the $\mathrm{KOH}$ mount showed fungal elements

bony erosion, erosion of the lamina papyracea and frank orbital invasion. Associated signs would include a thickening and lateral displacement of the medial rectus muscle 


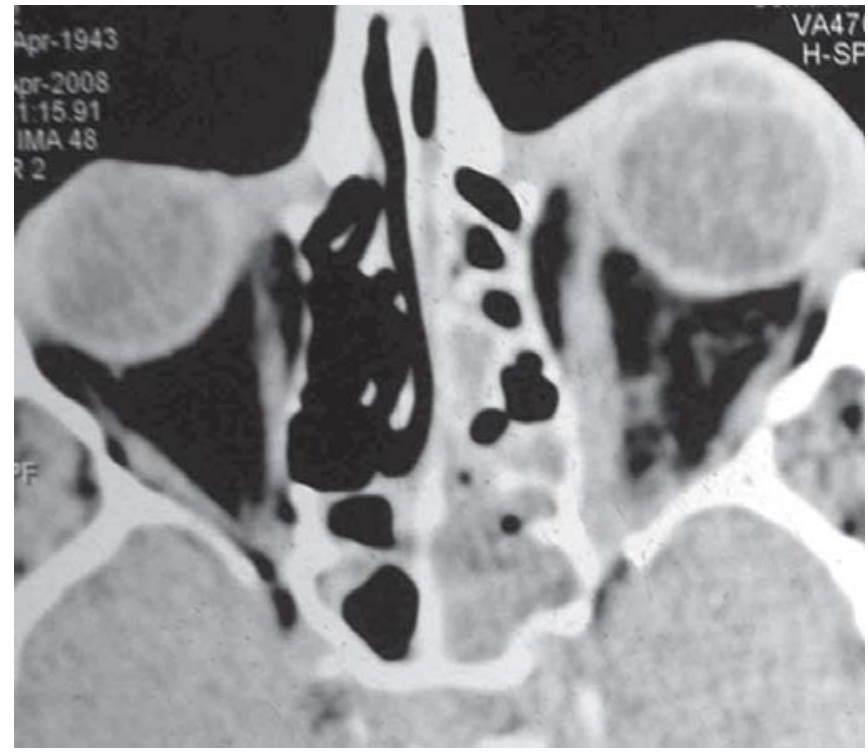

FIGURE 18: Axial contrast enhanced CT - PNS of a patient with left sided invasive fungal sinusitis, showing left proptosis and thickening of the left medial rectus muscle

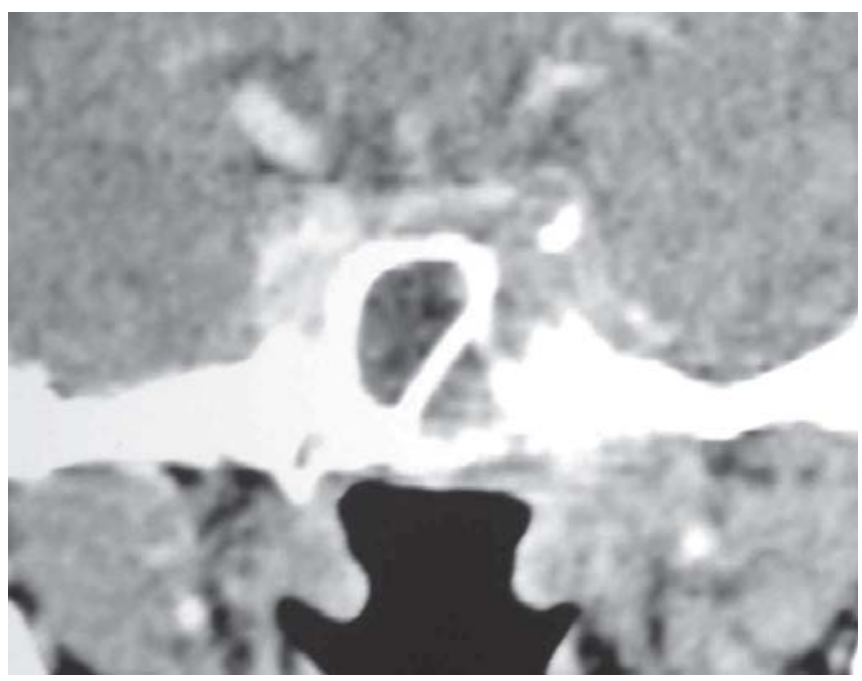

FIGURE 20: Coronal contrast enhanced CT - PNS showing left cavernous sinus thrombosis (nonuptake of contrast) in a patient with invasive fungal rhinosinusitis

(Fig. 18), which usually indicates orbital spread from the adjacent ethmoid sinuses. ${ }^{14,15}$ The optic nerve may also be thickened (Fig. 19). Nonenhancement of the superior ophthalmic artery and vein, which is related to vasculitis and thrombosis may represent rhino-orbito-cerebral zygomycosis. ${ }^{15}$ In cases with orbital involvement, with disease in the region of the orbital apex, the chances of

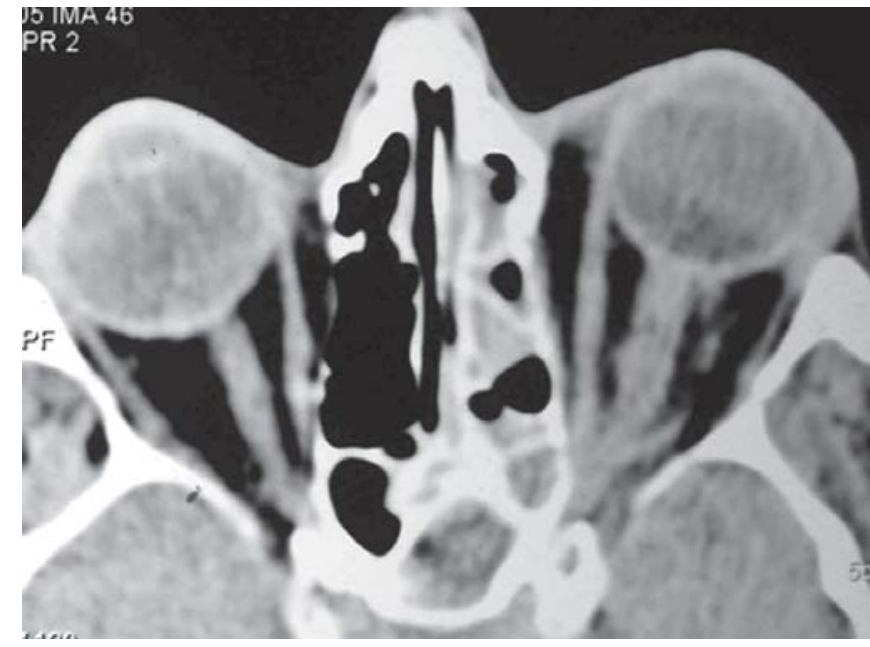

FIGURE 19: Axial contrast enhanced CT - PNS of a patient with left sided invasive fungal sinusitis, showing left proptosis and thickening of the left optic nerve

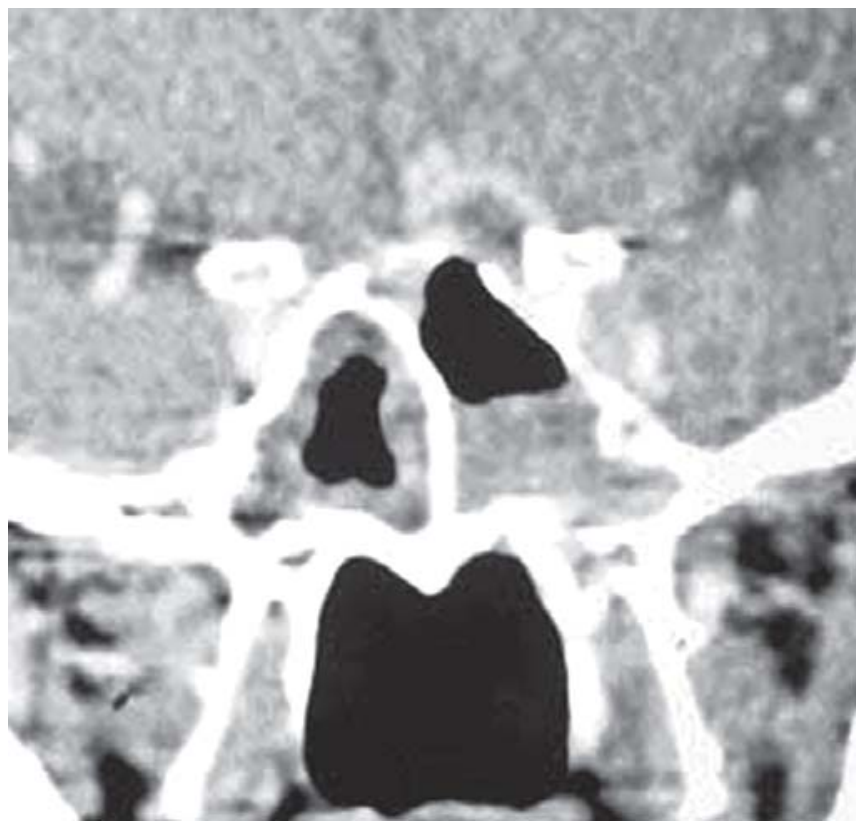

FIGURE 21: Coronal contrast enhanced CT - PNS showing a left parasellar mycotic abscess in the same patient (Fig. 20)

intracranial spread are high, since it forms a conduit for perineural and perivascular spread and it compromises the blood supply of the orbit which traverses and also branches within the region of the orbital apex, thus not allowing for systemic antifungals to reach the orbit. Such conditions would warrant an urgent orbital exenteration, so as to prevent intracranial spread; thus the site of fungal orbital invasion 


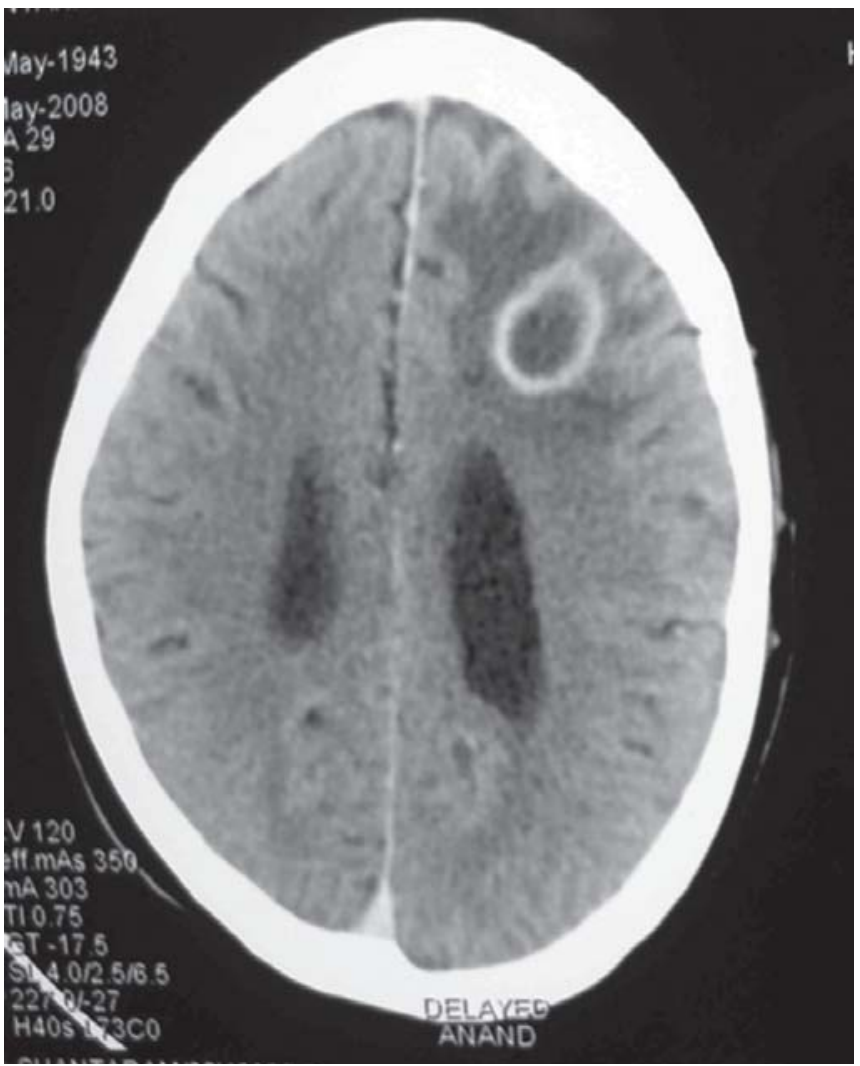

FIGURE 22: Axial contrast enhanced CT brain, showing a left cerebral mycotic abscess, with peripheral ring enhancement and perilesional tissue edema, in the same patient (Fig. 20)

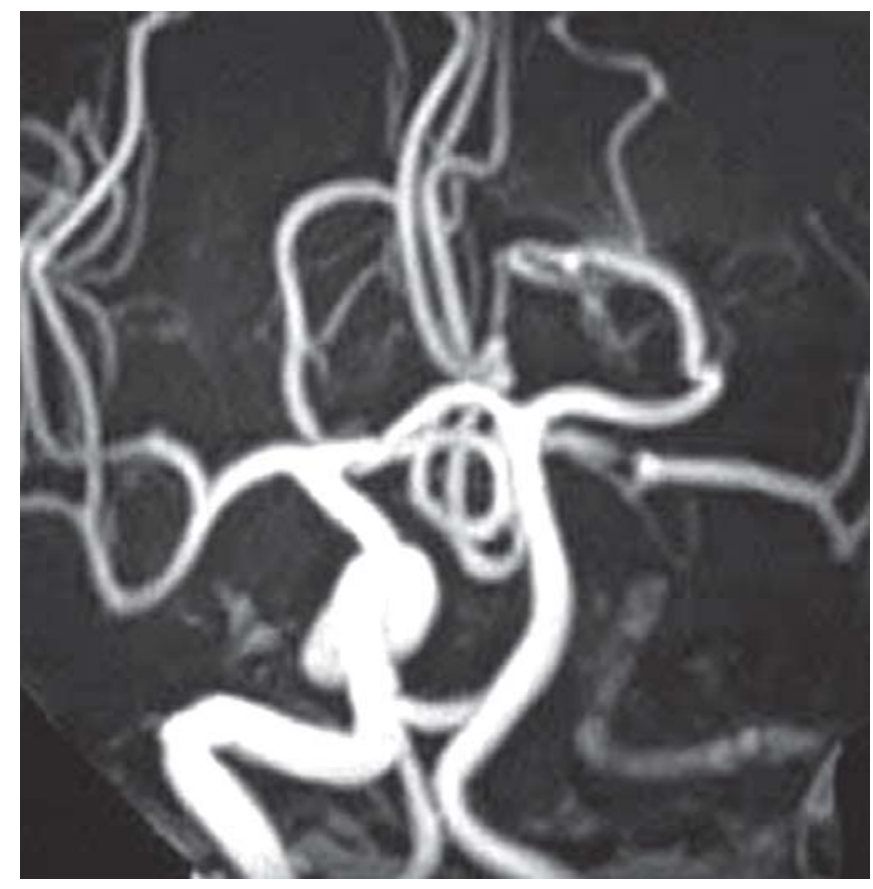

FIGURE 24: MR angiogram in the same patient as in Fig. 20, showing nonuptake of contrast in the left internal carotid artery, due to thrombosis

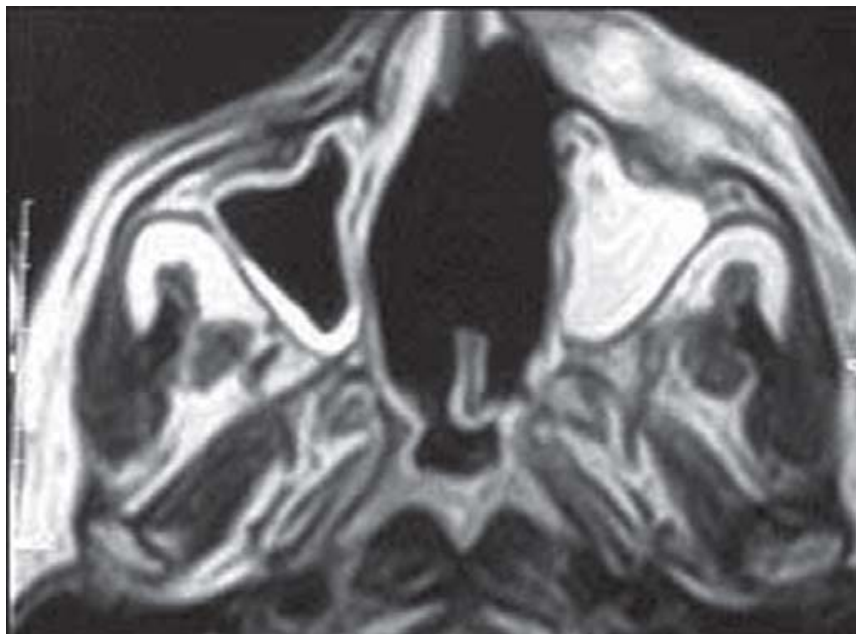

FIGURE 23: Axial T2 MR - PNS showing signal void in the nasal cavity and ethmoids with erosion of the nasal septum, and secondary maxillary sinusitis

could also indicate the prognosis, and help in tailoring surgical treatment.

Cavernous sinus thrombosis and other intracranial complications can also be detected on contrast enhanced CT scans (Figs 20 to 22).

\section{MRI Findings}

The MR signal is variable on T1 and T2 weighted images, because of varying amounts of edema and fungal elements. ${ }^{11}$ The secondary bacterial sinusitis shall show bright signals, especially on T2 images, while the actual fungal invasion would resemble hypoattenuated signals on $\mathrm{T} 1$ and void signals on T2 (Fig. 23). It is also helpful in detecting intracranial (Fig. 24) and orbital spread of the disease, and also to detect dural and periorbital thickening or invasion, indicating their involvement.

\section{REFERENCES}

1. Gillespie MB, Huchton DM, O’Malley BW. Role of middle turbinate biopsy in the diagnosis of fulminant invasive fungal rhinosinusitis. Laryngoscope 2000;110:1832-36.

2. DelGaudio JM, Swain RE Jr, Kingdom TT, et al. Computed Tomographic findings in patients with invasive fungal sinusitis. Arch Otolaryngol Head Neck Surg 2003;129:236-40.

3. Aygun M, Zinreich SJ. Imaging for Function Endoscopic Sinus Surgery. Otolaryngol Clin N Am 2006;39:403-16.

4. Schubert MS. Allergic Fungal Sinusitis. Otolaryngol Clin N Am 2004;37:301-26.

5. Mukherji SK, Figueroa R, Ginsberg LE, et al. Allergic Fungal Sinusitis: CT findings. Radiology 1998;207:417-22.

6. Nussenbaum B, Marple BF, Schwade ND. Characteristics of bone erosion in allergic fungal sinusitis. Otolaryngol Head Neck Surg 2001;124:150-54. 
7. Marple BF. Allergic fungal rhinosinusitis: A review of clinical manifestations and current treatment strategies. ISHAM, Medical Mycology 2006;44:5277-84.

8. Pagella F. Paranasal sinus fungal ball: Diagnosis and management. Pagella F, Matti E, De Bernardi F, Semino L, Cavanna C, Marone P, Farina C, Castelnuovo P. Mycoses 2007;50:451-56.

9. Daudia A, Jones NS. Advances in management of paranasal sinus Aspergillosis. J Laryngol Otol 2007;1-5.

10. Oyarzabal MF, Chevretton EB. Semi-invasive allergic Aspergillosis of the paranasal sinuses. J Laryngol Otol 2000;114:290-92.
11. Howells RC, Ramadan HH. Usefulness of computed tomography and magnetic resonance in fulminant invasive fungal rhinosinusitis. Am J Rhinol 2001;15:255-61.

12. Bransetter BF, Weissman JL. Role of MR and CT in the Paranasal Sinuses. Otolarygol Clin N Am 2005;38:1279-99.

13. Manning SC, Merkel M, Kriesel K, et al. Computed tomography and magnetic resonance diagnosis of allergic fungal sinusitis. Laryngoscope 1997;107:170-76.

14. Keefe MO, Haining WM, Young JDH, Guthrie W. Orbital mucormycosis wit survival. Br J Ophthal 1986;70:634-36.

15. Thomas PA. Current perspectives on ophthalmic mycoses. Clin Microbio Rev 2003;16(4):730-97. 